DOI: 10.1109/PIMRC.2016.7794936

\title{
Handover Implementation in a 5G SDN-based Mobile Network Architecture
}

\author{
Jonathan Prados-Garzon, Oscar Adamuz-Hinojosa, Pablo Ameigeiras, Juan J. Ramos-Munoz, \\ Pilar Andres-Maldonado, Juan M. Lopez-Soler \\ Department of Signal Theory, Telematics, and Communications \\ University of Granada \\ Granada, Spain \\ Emails: jpg@ugr.es, oscar93rah@correo.ugr.es,pameigeiras@ugr.es,jjramos@ugr.es,pam91@correo.ugr.es, juanma@ugr.es
}

\begin{abstract}
Requirements for $5 \mathrm{G}$ mobile networks includes a higher flexibility, scalability, cost effectiveness and energy efficiency. Towards these goals, Software Defined Networking (SDN) and Network Functions Virtualization have been adopted in recent proposals for future mobile networks architectures because they are considered critical technologies for 5G. In this paper, we propose an X2-based handover implementation in an SDNbased and partially virtualized LTE architecture. Moreover, the architecture considered operates at link level, which provides lower latency and higher scalability. In our implementation, we use MPLS tunnels for user plane instead of GTP-U protocol, which introduces a significant overhead. To verify the correct operation of our system, we developed a simulator. It implements the messages exchange and processing of the primary network entities. Using this tool we measured the handover preparation and completion times, whose estimated values were roughly 6.94 $\mathrm{ms}$ and $8.31 \mathrm{~ms}$, respectively, according to our experimental setup. These latencies meet the expected requirements concerning control plane delay budgets for $5 \mathrm{G}$ networks.
\end{abstract}

Index Terms-Handover, 5G, SDN, NFV, virtualized LTE/EPC.

\section{INTRODUCTION}

5G mobile networks are expected to be an unprecedented revolution in broadband wireless communications that will have an impact on every aspect of our society. The trends that motivate the definition of $5 \mathrm{G}$ technology are the following [1]:

- The explosive growth of mobile data traffic, which will increase more than 200-fold between 2010 and 2020.

- The increasing adoption of the Internet of Things (IoT), whose number of connections will reach 7 billion in 2020.

- The continuous emergence of new services (e.g., 3D ultra-high definition video, mobile cloud, mobile health, augmented reality, Tactile Internet applications) and application scenarios (e.g., ultra-dense and high speed moving scenarios).

Requirements for $5 \mathrm{G}$ mobile networks include a higher flexibility and scalability of the network, and an x100 increase in cost effectiveness and energy efficiency compared to its predecessor, the Long Term Evolution (LTE) technology [1]. Toward these goals, new paradigms such as Software Defined Networking (SDN) and Network Functions Virtualization (NFV) have been adopted in recent proposals for mobile network architectures [2] [3] [4] [5], and they are expected to play a vital role in $5 \mathrm{G}$ networks.
On the one hand, the SDN paradigm decouples control and user planes. In SDN, the control plane consists of a logically centralized controller implemented in software that controls a set of low-cost and simple network devices that make up the user plane. The controller can configure the forwarding tables and monitor packet statistics of the user plane switches using the OpenFlow (OF) protocol [6]. On the other hand, NFV offers the operators the possibility of running the network functions on industry standard high-volume servers instead of using expensive, special purpose, and vendor-dependent hardware [4].

In this work, we consider a partially virtualized architecture for $5 \mathrm{G}$ networks, which is the same as the one proposed in [2]. This architecture is based on SDN and NFV and consists of three hierarchical levels: the access cloud (AC), the regional distributed cloud (RC), and the national centralized cloud (NC) [2]. We concentrate on the AC mobility support and assume that the LTE control plane (CP) remains unchanged, but it is the user plane (UP) what is changed. Consequently, we assume the same X2-based Handover (HO) procedure as for LTE standard [7]. In this approach, all the HO procedures at the $\mathrm{AC}$ are unnoticed by the $\mathrm{RC}$ network (i.e., $\mathrm{RC}$ signaling workload offloading). Moreover, SDN allows us to remove the GTP-U protocol, which introduces a significant overhead at each UP packet.

This work aims at assessing the impacts of the mobility support on an SDN-based architecture for 5G networks and evaluating the handover procedure execution time in this architecture. To achieve these goals, we developed a simulator of the LTE X2-based HO procedure in an SDN-based architecture. It implements the UP and the messages exchange and processing of the main network entities. Besides, it simulates the transmission, propagation and processing delays of the network. Using this tool we checked the correct operation of the $\mathrm{HO}$ procedure in an SDN-based architecture. We also computed the HO preparation and completion times, whose estimated values were roughly $6.94 \mathrm{~ms}$ and $8.31 \mathrm{~ms}$, respectively, according to our experimental setup.

The main contribution of this paper is a proposal for a Handover implementation in an SDN-based and partially virtualized LTE network. Although some issues must be address to provide mobility support in this context, our solution has 
a negligible impact on the LTE CP. We describe this implementation and the operation of the system. Additionally, we implemented our solution and validated that it works properly. Finally, we assessed the handover procedure execution time of our solution.

The rest of the paper is organized as follows. Section II reviews the relevant literature. Sections III and IV describe the system architecture and the handover process considered in this work, respectively. Section V presents the impacts on the architecture brought about by the HO procedure implementation. Additionally, the HO implementation details are included. The experimental setup along with our results and its discussion are included in Section VI. Finally, Section VII draws the main conclusions.

\section{RELATED WORKS}

The adoption of SDN and NFV paradigms is expected to bring substantial benefits to future mobile networks regarding scalability, flexibility, costs, and energy consumption. For this reason, recent architecture proposals for such networks have made use of these paradigms. However, according to the authors in [3] the integration of SDN and NFV in Mobile Networks should be progressive. That is to favor the migration from legacy networks. To that end, they propose a 3-step migration scheme for the adoption of SDN and NFV technologies. In the fist step, the network entities are virtualized. In the second phase, SDN paradigm is included, while still maintaining legacy nodes. In the last step, the network is fully SDN-compliant, and GTP tunneling and legacy nodes at the UP of the core network such as Serving Gateway (S-GW) are removed. We consider the third step case to define our solution.

Several works have proposed solutions for the mobility support in SDN-based mobile networks. The proposal in [8] aims at removing the use GTP-U protocol at UP, while it preserves the $3 \mathrm{GPP}$ CP untouched. Unlike our proposal, this solution considers the use of legacy nodes (e.g., S-GW) at UP. Therefore, it belongs to the second step of the migration process. In [9], the authors discuss how to apply SDN in mobile networks to efficiently handle mobility. Specifically, they address problems related to mobility management like preservation of session continuity and scalability of HOs in dense scenarios. In addition, they consider and study three approaches for the SDN controller implementation (e.g., centralized, semi-centralized and hierarchical). The authors in [10] propose an SDN mobility management architecture and evaluate handover execution time by using a prototype of their architecture. They concentrate on defining extensions to the OpenFlow protocol for accomplishing S5/S8 functionality without introducing changes to the rest of the standard 3GPP defined interfaces [10].

\section{SYStEM ARChITECTURE}

In this work, we consider an envisioned future $5 \mathrm{G}$ mobile network for the Access Cloud (AC) that follows a partial virtualization model with an SDN-based UP. That is, only

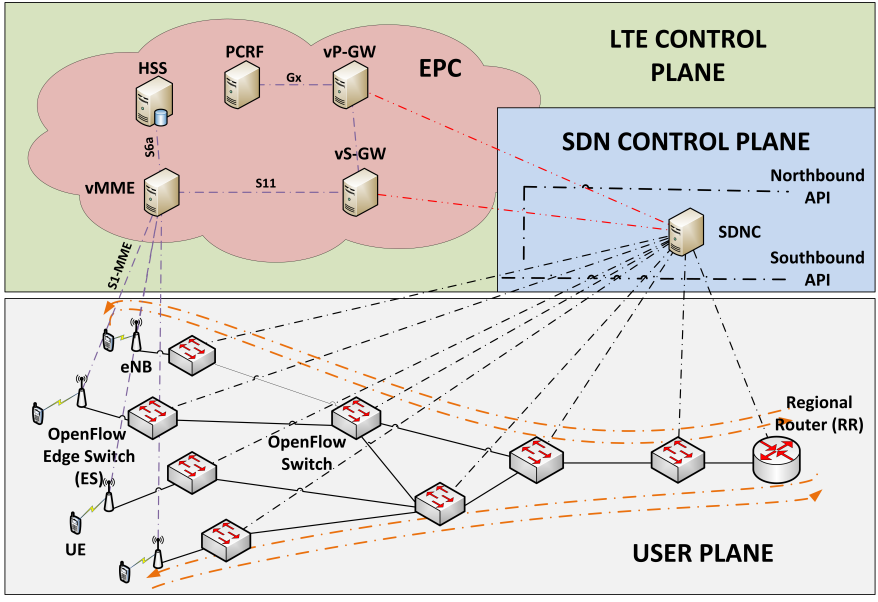

Fig. 1. System Architecture.

the LTE CP functional entities (i.e., LTE control plane) are implemented as Virtualized Network Functions (VNFs) running in a logically centralized data center, whereas the UP consists of OF commodity switches distributed in the network [4]. Specifically, the AC architecture considered is the same as the one proposed in [2] and the LTE CP remains unchanged [7] (see Fig. 1).

We consider that the design of the virtualized LTE control network entities (e.g., vMME, vS-GW, and vP-GW) follow a 1:N mapping architectural option [4] [11]. Thus, they are split into 3 logical components: front-end (FE), service logic (SL), and state database (SDB). The FE is implemented with an OF switch and acts as a communication interface with other entities of the network and balance the load among several SLs, which implement the processing of the different control messages. The SDB stores the user session state making the SLs stateless. Therefore, the number SLs can grow without affecting on in-session users. Moreover, the virtualized entity is seen like a single component from the rest of the network.

There is an SDN Controller (SDNC) (i.e., SDN control plane) for the $\mathrm{AC}$ that acts as an interface between the LTE CP and the UP. For instance, the vS-GW interacts with the SDNC through the Northbound Application Programming Interface (API). Accordingly, vS-GW can be seen as network applications running on the top of the SDNC. The SDNC controls all the UP switches through the Southbound API (i.e., OF protocol) by signaling the OF table entries.

Please note that to virtualize the S-GW and Packet Data Network Gateway (P-GW), their functionality must be split into control and user planes. For example, the vS-GW implements the CP capability, and it must be extended to interact with the SDNC through the Northbound API. In contrast, the S-GW UP functionality is carried out by the Regional Router (RR), which is an OF switch, acts as the AC mobility anchor, and provides access to external networks.

Like in LTE, Users Equipments (UEs) are the terminals which allow each user to connect to the network via the 
eNodeBs (eNBs). The UEs run the users' applications which generate or consume UP traffic. This process also triggers the LTE control procedures such as Handover, Service Request or S1 Release [7]. The users move freely along the coverage area of the Radio Access Network (RAN). Assuming that each eNodeB serves only one cell, an HO procedure takes place when a user performs a cell change while being in EMMRegistered and ECM/RRC-Connected States.

\section{Handover (HO) Procedure}

Here we describe the $\mathrm{HO}$ procedure performed when a UE is in EMM-Registered and ECM/RRC-Connected States and moves from the coverage area of a Source eNB (SeNB) to the one of a Target eNB (TeNB) within the AC (Fig. 2). We consider the same $\mathrm{HO}$ procedure and the scenario as the $\mathrm{X} 2$ based HO of LTE [7], assuming that MME and S-GW are not relocated. We also suppose that $\mathrm{HO}$ processes associated with the radio interface protocols are the same as the 3GPP-based LTE system.

The main steps of the $\mathrm{HO}$ procedure are listed bellow (see Fig. 2):

- Firstly, when the signal level from TeNB overcomes a threshold, the UE sends a Measurement Report to the SeNB. Then, the SeNB makes the HO decision and forwards a Handover Request message to the TeNB.

- Secondly, the TeNB executes an admission control procedure to determine whether it has available resources to support the incoming UE. If the TeNB admits the UE, it acknowledges the $\mathrm{HO}$ sending Handover ACK message to the SeNB, which in turn confirms it to the UE.

- At this point, the SeNB begins a redirection procedure forwarding buffered and incoming downlink frames for the UE to the TeNB. To support lossless HO, the SeNB can provide the sequence numbers of the forwarded frames through the Handover Context Information message. At the same time, the $\mathrm{HO}$ interruption time takes place, where the UE carries out a synchronization process with the TeNB. During this period the UE cannot send or receive any data frame. Once the UE synchronizes with the TeNB, it sends the Handover Confirmation message to the TeNB. From this time on, the TeNB can directly send UE uplink frames to the RR.

- Next, the TeNB sends a Path Switch Request message to vMME to notify that the UE has performed an eNB change. After receiving this message, the vMME informs the vS-GW that the downlink S1 bearer has been switched, and asks to switch the bearer path accordingly by sending a Modify Bearer Request message. After processing this message, the $\mathrm{vS}-\mathrm{GW}$ sends an Update User Plane Request to the SDNC to modify the corresponding flow table entry of the RR, which acts as the mobility anchor. Once the SDNC concludes the operation, it generates the Update User Plane Reply, which is sent to the vS-GW to confirm the UP update. The vS-GW in turn acknowledges the Path Modify Bearer Request with Path Modify Bearer Response message.
- Finally, the vMME notifies the TeNB that the new path has been established with a Path Switch Request ACK message. The TeNB, in turn, sends a UE Context Release message to the source eNB. Now the source eNB can release radio and $\mathrm{CP}$ resources allocated for the UE and the $\mathrm{HO}$ procedure concludes.

\section{SDN-BASED MOBILITY SUPPORT}

This section addresses the implementation of the LTE X2based Handover procedure in a partially virtualized LTE network. Even though our solution uses the same CP as LTE networks, the mobility support in such architecture entails some changes such as adding new processes and extension of functionality of the some LTE network entities. Additionally, the operation and update of the UP during HO procedure widely differ from LTE networks. Next, we describe these modifications and differences.

\section{A. User plane operation}

For simplicity, we assumed that all the data traffic flows are north-south, i.e. all data traffic passes through the Regional Router (RR), which acts as a gateway towards external networks. There is an OF switch for each eNB that interconnects the eNB with the backhaul network (BN) (see Fig. 1), referred as edge switch (ES). The ESs and the RR are hereafter referred to as the Edge Network Elements (ENEs) [2].

At UP we use MPLS tunnels, which are handled by the SDNC, instead of GTP tunnels. The MPLS tunnels introduce only 4 bytes of overhead to every UP packet, while GTP$\mathrm{U}$ protocol adds an overhead of 36 bytes (IPv4). When a UE triggers a service request procedure, the SDNC creates a tunnel for the UE simply by adding an entry in the RR flow table. Consequently, the RR will have an OF entry per each user to which packets have been destined recently (i.e., users in ECM/RRC-Connected State). These OF table entries in the RR are deleted during service releases and detach control procedures to avoid scalability issues (especially for MachineTo-Machine devices).

For every incoming UP packet, the ingress ENE pushes a multiprotocol label switching (MPLS) header with a label field. The label is associated with the egress ENE where the packet will be forwarded to. The match field used for table lookup is the destination IP. The OF actions applied to the frame are push-MPLS and output [6]. The output action is configured to forward the packet on the port towards the BN. At every BN switch, the UP packet is processed and forwarded according to the OF table entry that uses the MPLS label as match field. The egress ENE simply pops the MPLS header and delivers the packet. For downlink data traffic the ingress ENE will be the RR and the egress ENE will be any ES or the other way around in case of uplink data traffic. Please note that other tags instead of MPLS label could be used for routing purposes at the $\mathrm{BN}$.

Finally, it shall be noted that the SDNC is in charge to allocate MPLS labels for each ENE during network configuration. A routing application running on the SDNC computes and 


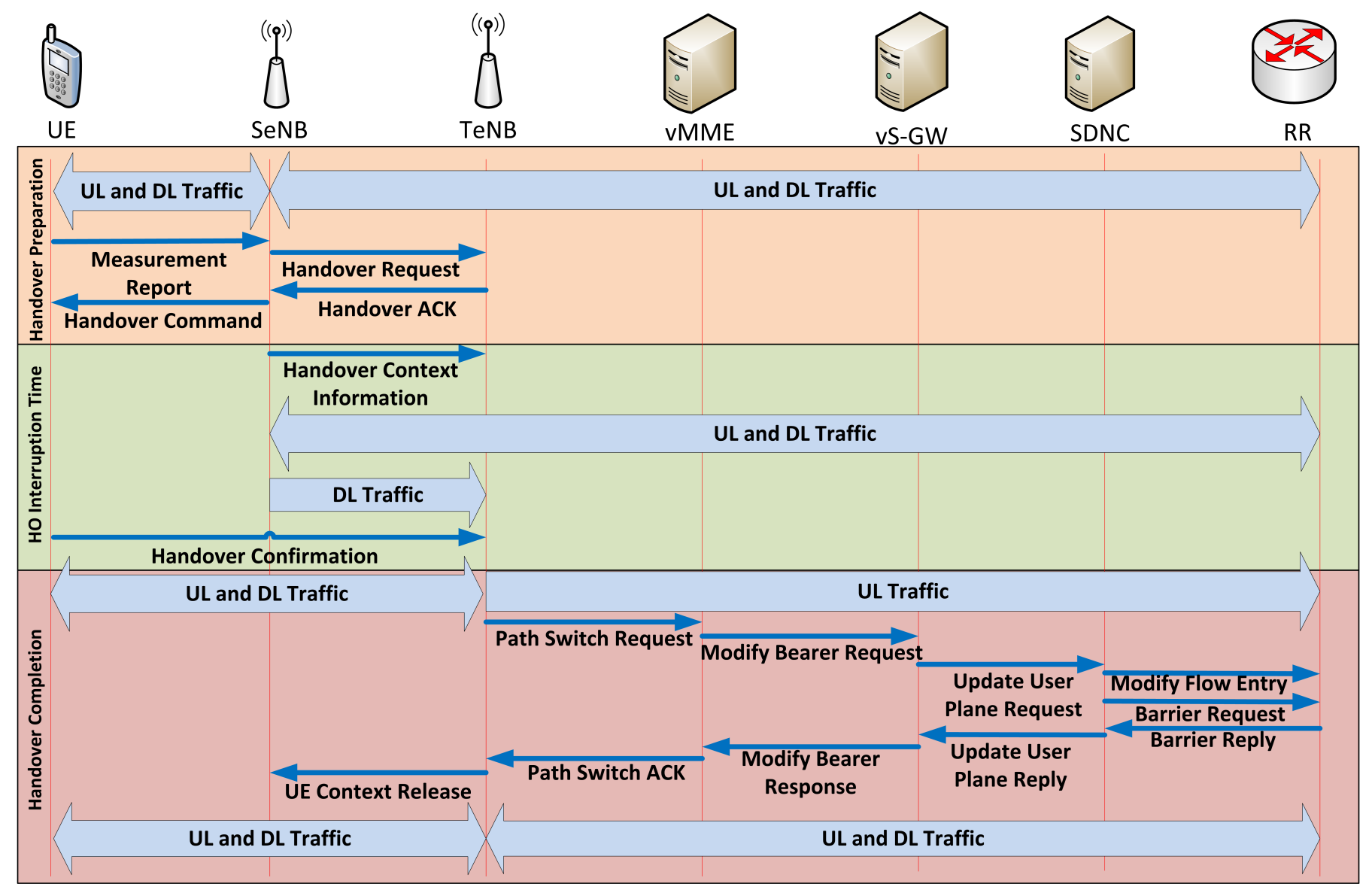

Fig. 2. Openflow-based Handover procedure.

installs the routes for the BN. This application also monitors the links state of the BN.

\section{B. X2 interface}

The eNBs are interconnected with each other through X2 interface [7]. The same network infrastructure as UP is used to support the X2 interface. Each eNB stores the Neighbour Information Table (NIT) that relates the Physical Cell Identifier (PCI), Evolved Cell Global Identifier (ECGI) and the IP address of its neighbouring eNBs. On the one hand, the eNB might use the LTE Automatic Neighbour Relation Function (ANRF) to discover automatically its adjacent eNBs and to establish the corresponding relation between ECGI and PCI [12]. On the other hand, the eNB might learn the IP addresses of its neighbouring eNBs by requesting them directly to the SDNC. The SDNC needs to store a table, named the Network Information Base (NIB), that contains all the identifiers allocated for all of the network entities (e.g., PCIs, ECGIs, IP addresses, MPLS labels,...).

Whenever an eNB requests an IP address of a neighbouring eNB, the SDNC reply this message and adds an entry in the flow table of the ES associated with the requester eNB. This entry uses the destination IP address as match field and its value is fixed to the neighbouring eNB IP address. Like
UP case, the actions specified for this entry are push MPLS header and forwards the packet to the $\mathrm{BN}$. In this way, the reachability between neighbouring eNBs is enabled to support $\mathrm{X} 2$ interfaces.

\section{HO procedure considerations}

As described in Section III, the S-GW functionality must be split into control and user planes. The control functionality is implemented by the vS-GW, while the UP functionality is implemented by the RR. Additionally, the control functionality at the vS-GW needs to be extended to allow the interaction with the SDNC through the northbound API. In our case, the vS-GW is able to generate the Update User Plane Request message (see Fig. 2), which contains the target eNB's ECGI and the IP address of the UE performing the handover. This entity also process the Update User Plane Reply message, which is a confirmation that the path switch have been performed correctly at UP.

The SDNC is in charge to carry out the UP update in the HO procedure. That is to modify the corresponding entry in the Regional Router flow table. When the SDNC receives Update User Plane Request message, it sends an OF Modify Flow Entry message to update the corresponding entry at the RR. In addition, the SDNC sends an OF Barrier Request message 
to the Regional Router. That is to receive notification from the $\mathrm{RR}$ when the operation is completed.

The packets destined to SDNC are not associated with any flow at any OF switch of the network. Consequently, they will be encapsulated in an OF Packet IN message [6] (i.e., our default action configured in case of OF table miss-match) and directly sent to the SDNC via the OpenFlow interface. The control messages sent to the virtualized control LTE entities (e.g., vMME and vS-GW) do not need to passing through the SDNC controller. That is because MPLS tunnels are also employed to support connectivity between the primary network entities. Thus, the virtualized control entities could be considered as ENEs.

Finally, the target eNB needs to buffer the downlink (DL) data packets sent by the source eNB during the HO interruption time.

\section{RESUlTS AND DISCUSSION}

In this section, we describe our experimental setup and carry out an assessment of the HO procedure execution time.

\section{A. Experimental Setup}

We developed a simulator of the X2-based LTE HO procedure in the SDN-based architecture described in this work. It implements the UP, and the messages exchange and processing of the different network nodes. Furthermore, it simulates the transmission and propagation delays for each link (see Fig. 3 and Table I). This simulator was implemented within the ns-3 environment [13], using the OpenFlow module. By using it, we verified that the handover procedure works correctly. Moreover, we measured the $\mathrm{HO}$ execution time of our proposal for a given scenario.

The simulation scenario considers a tree topology with two layers as shown in Fig. 3 (the ESs are not depicted in the Figure). We assumed that control and user planes share the same network infrastructure, and there is not differentiated treatment for the control messages.

Regarding the radio interface, we considered a constant transmission delay of $2 \mathrm{~ms}$ for each control packet (e.g., Measurement report and Handover Command) [14]. We also supposed that every eNB has enough radio resources to support the data rate demanded by all its attached UE. The UE measurement reports are triggered when the LTE event A3 occurs, i.e. the Reference Signal Received Power (RSRP) of the TeNB becomes stronger than the RSRP of the SeNB by an offset.

As can be seen from Fig. 3, two kinds of handover cases, which are named as Intra-Switch Handover and Inter-Switch Handover, might appear in our scenario. The first happens when SeNB and TeNB are connected to the same BN switch (Level 1), whereas the second occurs when they are connected to different $\mathrm{BN}$ switches. The $\mathrm{HO}$ execution time will be slightly higher for Inter-Switch HO case. Considering our scenario, we include the probability of occurrence of such HO cases in Table I.

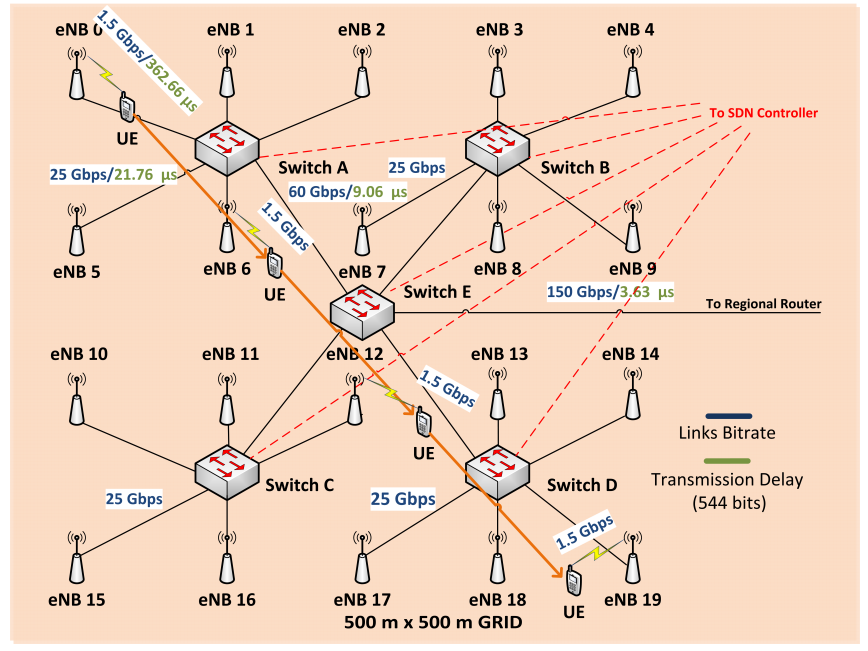

Fig. 3. Experimental topology

TABLE I

PARAMETERS CONFIGURATION

\begin{tabular}{|c|c|}
\hline \multicolumn{2}{|c|}{ Network topology } \\
\hline eNBs layout & Regular Grid 500 m x 500 m [15] \\
\hline eNB coverage area & $100 \mathrm{~m} \times 125 \mathrm{~m}$ \\
\hline Number of eNBs & 20 \\
\hline $\begin{array}{l}\text { Position of the eNB } \\
i \in\{0, \ldots, 19\}\end{array}$ & {$[50+(i \% 5) \cdot 100,62.5+\lfloor i / 5\rfloor \cdot 125]$} \\
\hline Number of UEs & 100 \\
\hline Backhaul topology & Tree (2 Levels) [16] \\
\hline $\begin{array}{l}\text { Position of the BN } \\
\text { switches }\end{array}$ & $\begin{array}{l}\text { Square vertices and center }(250,250) \text {. } \\
\text { Side length: } 250 \mathrm{~m}\end{array}$ \\
\hline RR position & $25 \mathrm{Km}$ from Switch $\mathrm{E}$ \\
\hline \multicolumn{2}{|r|}{ UE Mobility } \\
\hline Mobility model & Fluid-flow model [17] \\
\hline UE speed & $6 \mathrm{~m} / \mathrm{s}$ \\
\hline \multicolumn{2}{|c|}{ Traffic model (UP) } \\
\hline Traffic model type & ON-OFF model \\
\hline ON and OFF periods & Uniformly distributed $(0,1)$ seconds \\
\hline \multicolumn{2}{|c|}{ Processing delays } \\
\hline$\overline{\mathrm{eNB}}$ & $960 \mu s[18]$ \\
\hline $\begin{array}{l}\text { OF Switch without } \\
\text { modifying headers }\end{array}$ & $5 \mu s[19][20]$ \\
\hline $\begin{array}{l}\text { OF Switch modifying } \\
\text { headers }\end{array}$ & $10 \mu s[21]$ \\
\hline SDN Controller & $3 m s[22]$ \\
\hline vSGW & $1 \mathrm{~ms}$ \\
\hline vMME & $1 m s[11]$ \\
\hline Regional Router & $100 \mu s$ \\
\hline \multicolumn{2}{|c|}{ Propagation delays } \\
\hline $\begin{array}{l}\text { Speed of light in wireless } \\
\text { and wired links }\end{array}$ & $300000 \mathrm{~km} / \mathrm{s}$ \\
\hline \multicolumn{2}{|c|}{ Handover measurement reports } \\
\hline Event type & $\begin{aligned} \mathrm{A} 3 \text { with off set } & =2 d B \text { and } \\
\text { hysteresis } & =0 d B\end{aligned}$ \\
\hline Carrier frequency & $2.12 \mathrm{GHz}$ \\
\hline eNB Tx Power & $30 \mathrm{dBm}$ \\
\hline Path loss model & Friis \\
\hline \multicolumn{2}{|c|}{ Handover cases (probability) } \\
\hline Intra-Switch Handover & $80 / 126$ \\
\hline Inter-Switch Handover & $46 / 126$ \\
\hline
\end{tabular}




\section{B. Handover execution time}

Considering our experimental setup, we assessed the $\mathrm{HO}$ preparation and $\mathrm{HO}$ completion times for different UE traffic rates at UP (see Fig. 4). The HO execution time is the sum of these contributions and the $\mathrm{HO}$ interruption time. We assumed a constant $\mathrm{HO}$ interruption time time of $15 \mathrm{~ms}$ [18].We observed that $\mathrm{HO}$ preparation and completion times are almost constant for data traffic rates per UE up to $1 \mathrm{Gbps}$, with values $6.94 \mathrm{~ms}$ and $8.31 \mathrm{~ms}$ respectively. From this point on the HO execution time increases because the backhaul network begins to exhibit congestion. In other words, the time spent waiting in queues at $\mathrm{BN}$ rise.

According to [23], the CP delay budget for X2-based HO preparation and completion phases is $31 \mathrm{~ms}$. Also, it is expected that latency requirements for $5 \mathrm{G}$ networks will be two times more rigid in CP [24]. Based on the above, the delay obtained for HO preparation and completion phases (15.25 ms) meets the $\mathrm{CP}$ latency requirements for $5 \mathrm{G}$ networks.

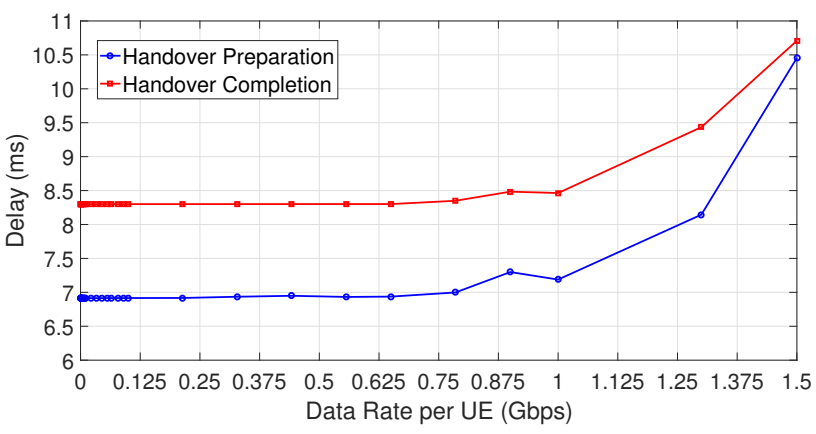

Fig. 4. Handover Execution Time.

\section{CONCLUSIONS}

In this paper, we propose and describe at length an implementation of the X2-based Handover procedure in a partially virtualized LTE network. We have developed a systemlevel simulator of this network within the ns-3 environment. This simulator includes an implementation of all the network entities, its messages exchange, the UP and the OpenFlow protocol. Using this tool we have verified that our implementation proposal works correctly, and we have assessed the Handover procedure execution time. Experimentally, we obtained a handover preparation time around $6.94 \mathrm{~ms}$, and a handover completion time around $8.31 \mathrm{~ms}$. These times fulfill the $\mathrm{CP}$ delay constraints of $5 \mathrm{G}$ networks.

\section{ACKNOWLEDGMENT}

This work is partially supported by the Spanish Ministry of Economy and Competitiveness and the European Regional Development Fund (project TIN2013-46223-P), and the Spanish Ministry of Education, Culture and Sport (FPU grant 13/04833).

\section{REFERENCES}

[1] $5 G$ Vision and Requirements, White paper, IMT-2020 (5G) Promotion Group, May 2014.

[2] P. Ameigeiras et al., "Link-level access cloud architecture design based on SDN for 5G networks," IEEE Network, vol. 29, no. 2, pp. 24-31, Mar. 2015.

[3] J. Costa-Requena et al., "SDN and NFV integration in generalized mobile network architecture," in Networks and Commun. (EuCNC), 2015 European Conf. on. IEEE, 2015, pp. 154-158.

[4] T. Taleb et al., "EASE: EPC as a service to ease mobile core network deployment over cloud," IEEE Network, vol. 29, no. 2, pp. 78-88, 2015.

[5] Y. Kyung et al., "Software defined service migration through legacy service integration into $4 \mathrm{G}$ networks and future evolutions," IEEE Commun. Mag., vol. 53, no. 9, pp. 108-114, Sept. 2015.

[6] OpenFlow Swicth Specification, version 1.4.0, Open Networking Foundation (ONF) TS-012, October 2013.

[7] General Packet Radio Service (GPRS) enhancements for Evolved Universal Terrestrial Radio Access Network (E-UTRAN) Access, 3GPP TS 23.401 Rel 12, 2014.

[8] J. Banik et al., "A Software Defined Semi Distributed Mobility Management System Based on Layer 2 Backhaul Network," in Veh. Technology Conf. (VTC Fall), 2015 IEEE 82nd, Sept. 2015, pp. 1-5.

[9] S. Kukliski et al., "Handover management in SDN-based mobile networks," in 2014 IEEE Globecom Workshops (GC Wkshps), Dec 2014, pp. 194-200.

[10] L. M. Contreras et al., "Software-Defined Mobility Management: Architecture Proposal and Future Directions," Mobile Networks and Applicat., pp. 1-11, 2016, article in Press. [Online]. Available: www.scopus.com

[11] J. Prados-Garzon et al., "Latency evaluation of a virtualized MME," in 2016 Wireless Days (WD), Mar. 2016, pp. 1-3.

[12] S. Sesia, I. Toufik, and M. Baker, LTE, The UMTS Long Term Evolution: From Theory to Practice. Wiley Publishing, 2009.

[13] G. F. Riley and T. R. Henderson, "The ns-3 network simulator," in Modeling and Tools for Network Simulation. Springer, 2010, pp. 15-34.

[14] D. Han et al., "Measurement and stochastic modeling of handover delay and interruption time of smartphone real-time applications on lte networks," IEEE Commun. Mag., vol. 53, no. 3, pp. 173-181, Mar. 2015.

[15] M. S. Guideline, "METIS Deliverable D6. 1 Simulation Guidelines," 2013.

[16] Ceragon, "Wireless Backhaul Topologies: Analyzing Backhaul Topology Strategies," pp. 1-15, August 2010. [Online]. Available: http://www.winncom.com/images/stories/Ceragon_Wireless_ Backhaul_Topologies_WP.pdf

[17] R. R. Roy, Handbook of mobile ad hoc networks for mobility models. Springer Science \& Business Media, 2010.

[18] D. Singhal et al., "LTE-advanced: handover interruption time analysis for IMT-A evaluation," in Signal Process., Communication, Computing and Networking Technologies (ICSCCN), 2011 Int. Conf. on. IEEE, 2011, pp. 81-85.

[19] T. Liu, "Implementing OpenFlow switch using FPGA based platform," in Dept. of Telematics, Norwegian University of Sci. and Technology, June 2014.

[20] F. Dürr et al., "Comparing the Forwarding Latency of OpenFlow Hardware and Software Switches," Tech. Rep. Comput. Sci. 2014/04, University of Stuttgart, Faculty of Comput. Sci., Elect. Eng., and Inform. Technology, Germany, University of Stuttgart, Inst. of Parallel and Distributed Syst., Distributed Syste., Tech. Rep., 2014.

[21] H. Uppal and D. Brandon, "OpenFlow based load balancing," CSE561: Networking Project Report, University of Washington, 2010.

[22] A. Tootoonchian et al., "On Controller Performance in Software-Defined Networks," in Presented as part of the 2nd USENIX Workshop on Hot Topics in Manage. of Internet, Cloud, and Enterprise Networks and Services, 2012.

[23] Z. Li and M. Wilson, "User plane and control plane separation framework for home base stations," Fujitsu Scientific and Tech. J., vol. 46, no. 1, pp. 79-86, 2010.

[24] F. Hu, Opportunities in $5 G$ Networks: A Research and Development Perspective. CRC Press, 2016. 